\title{
Asymmetry and Contradiction of Mirror Misalignments Influence of Off-Axial Cylindrical Hybrid Resonator
}

\author{
N. WANG*, X.X. Li AND H.F. ZHU \\ College of Science, China University of Petroleum (Huadong) \\ Qingdao, Shandong, 266580, People's Republic of China
}

(Received November 9, 2012)

\begin{abstract}
The output far-field intensity distributions of off-axial cylindrical hybrid resonator with different mirror misalignments are simulated by coordinate transformation fast Fourier-transform algorithm. The simulations showed that the mirror misalignments influence is different in anticlockwise and clockwise tilt direction. According to the calculation results, the output beam quality of off-axial unstable resonator is analyzed, from the aspects of $M^{2}$ value and power in the bucket curves. Furthermore, the analysis brings out an interesting contradiction that the beam quality changing evaluated by $M^{2}$ value is different from that by power in the bucket curves when the mirror misalignments occurred in anticlockwise direction. It demonstrated that the beam quality evaluation of off-axial unstable resonator should not be one-sided.
\end{abstract}

DOI: $10.12693 /$ APhysPolA.124.661

PACS: 42.55.Xi, 42.60.Da, 42.60.Jf

\section{Introduction}

The main problem of high power laser design is how to realize single mode operation, extract energy from the active medium effectively, and keep high beam quality. For high power continuous wave lasers with large size active medium, traditional unstable resonator with coaxial structure is usually used. But for slab laser, the gain medium has different size and pumped method in two orthogonal directions. The traditional structure is not beneficial to get good mode matching in two directions and utilize gain volume fully. By non-axial symmetric design, the cylindrical mirror cavity can be configured in different pumping and cooling conditions of two directions. Some gas $[1,2]$ and solid state $[3,4]$ slab lasers have successfully used confocal cylindrical hybrid resonator. Off-axial confocal configuration is very attractive because of its output beam properties, such as partially end-pumped slab laser [3], combining the hybrid resonator with partially end-pumping, high output power can be got with good beam quality [5-7], keeping the compact configuration simultaneously.

To design and build such resonator, it is necessary to have a detailed knowledge of mode properties. Sziklas and Siegman [8] use coordinate transformation fast Fourier transform (FFT) algorithm to calculate mode field of unstable resonator, and solve the Fourier spectrum aliasing problem of diverging or converging beam. Although the used resonator is co-axial and symmetric [8], the off-axial and asymmetric unstable resonator can be also calculated based on this method as our previous work [9].

*corresponding author; e-mail: qfwangning@aliyun.com
In this paper, the output beam properties of off-axial cylindrical hybrid resonator are simulated. By the simulation results, the asymmetry and contradiction of mirror misalignments influence are discussed. The analysis deepens the understanding of off-axial unstable resonator mode characters.

\section{Theoretical simulation method}

The typical off-axial cylindrical hybrid resonator [6] is showed as Fig. 1. In the horizontal direction an off-axial positive-branch confocal unstable cavity was formed by the concave and convex surfaces. In the vertical plane, a plane-plane resonator was configured. The slab crystal was put inside the cavity with large faces perpendicular to the cylindrical axes of the mirrors. The thermal distribution is quasi-one-dimensional because the large faces of crystal are cooled well. The stable-unstable hybrid resonator fully used the slab crystal structure by stable-unstable resonator combination. By the high-order mode restraining ability of unstable resonator, the nearly diffraction limit output is got in large size direction. In small size direction, the efficient and good quality laser oscillation is got by good mode matching between small pumped volume and small fundamental mode volume of end-pumped stable resonator.

In stable resonator direction, the output beam properties of plane-plane resonator are familiar to us. The study will be focused in unstable resonator direction. In this direction, coordinate transformation FFT algorithm [8] is used to solve the aliasing problem in the Fourier spectrum of usual diffraction fast Fourier-transform algorithm.

It has been demonstrated $[10,11]$ that the general character of "loaded resonator" modes is very little changed from that of "bare resonator" ones, the main difference being reduced amplitude of higher peaks. The media 


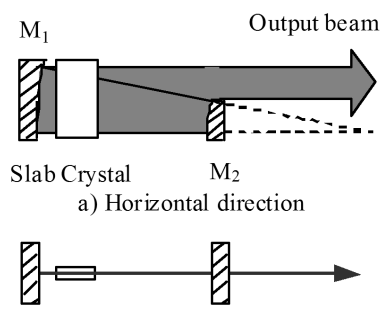

b) Vertical direction

Fig. 1. Diagram of off-axial cylindrical hybrid resonator.

gain is neglected at simulation. Because the thermal distribution is quasi-one-dimensional, the thermal lens effect is also neglected in unstable direction. When the wave has experienced round-trip many times in resonator, the phase distribution and amplitude distribution should be self-consistent. So the round-trip wave function transformations are shown as follows by the coordinate transformation FFT algorithm in Ref. [8]:

$$
\begin{aligned}
& u_{1}\left(x^{\prime}, y^{\prime}\right)=F^{-1}\left\{F \left[z_{0} \exp \left(-\mathrm{i} \frac{k y^{2}}{2 z_{0}}\right)\right.\right. \\
& \left.\left.\times \Psi_{0} P_{1} \mathrm{e}^{\mathrm{i} \pi \lambda\left(L \nu_{x^{\prime}}^{2}+M L \nu_{y^{\prime}}^{2}\right)}\right]\right\}, \\
& u_{2}\left(x^{\prime}, y^{\prime}\right)=F^{-1}\left\{F \left[\frac{1}{z_{0}+L} \exp \left(\mathrm{i} \frac{k y^{\prime 2}}{2\left(z_{0}+L\right)}\right)\right.\right. \\
& \left.\left.\times u_{1} P_{2} \exp \left(-\mathrm{i} \frac{k y^{\prime 2}}{2 f_{1}}\right) \mathrm{e}^{\mathrm{i} \pi \lambda L\left(\nu_{x^{\prime}}^{2}+\nu_{y^{\prime}}^{2}\right)}\right]\right\} .
\end{aligned}
$$

Here $u_{1}\left(x^{\prime}, y^{\prime}\right)$ and $u_{2}\left(x^{\prime}, y^{\prime}\right)$ are the wave function at cylindrical mirror $M_{1}$ and at cylindrical mirror $M_{2} ; P_{1}$ and $P_{2}$ are diaphragm functions of $M_{1}$ and $M_{2} ; F$ means the Fourier transform; $F^{-1}$ means inverse Fourier transform. $\Psi_{0}=A \exp \left(-\mathrm{i} \frac{k y^{2}}{2 f_{2}}\right)$, which is the reflected diverging wave function at the cylindrical mirror $M_{2} ; A$ is constant. For the confocal unstable positive-branch cavity, $f_{2}=-z_{0}, f_{1}=z_{0}+L$.

In unstable resonator direction, off-axis unstable resonator can be considered as half of coaxial unstable resonator. By adjusting diaphragm function and sampling points, the intensity distribution can be calculated. The output far-field distribution can be also got by self-consistent field distribution.

\section{Results and discussion}

In Fig. 2, the comparison of intensity distribution got by our simulation and in the experiments of Ref. [6] is shown. The simulation results evidently showed that our simulations correctly reconstructed the field distribution of extracted beam. The simulation is done by Matlab software programming. According to Refs. [6, 12], the front cylindrical mirror $M_{1}$, radii of curvature $R=$ $500 \mathrm{~mm}$; the back cylindrical mirror, radii of curvature

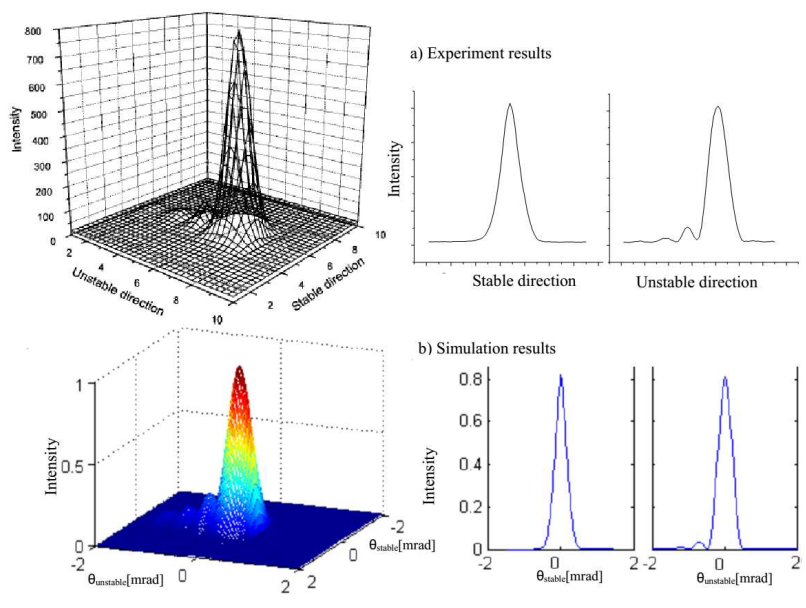

Fig. 2. Comparison of far-field distribution got by simulations with the beam profiles measured in experiments of Ref. [6]: (a) experiment results, (b) simulation results.

$R=-350 \mathrm{~mm}$; the thermal lens foci of stable resonator direction is $120 \mathrm{~mm}$.

In stable resonator direction, as the simulation results showed, its thermal stability is still good even if the thermal lens effect is very strong $(f=120 \mathrm{~mm})$, with the good output beam quality (calculated $M^{2}$ is 1.3 ). The output far-field intensity distributions of unstable resonator direction with different mirror misalignments are simulated in Fig. 3. Curve (a) is the calculated result when the mirror $M_{1}$ is not tilted. When the mirror is tilted in anticlockwise direction, curve (b) and (c) is respectively the far-field distribution of $10 \mu \mathrm{rad}$ mirror tilt and $30 \mu \mathrm{rad}$ mirror tilt. The far-field distribution with clockwise tilt is showed as curve (d), (e), (f), respectively with $-10 \mu \mathrm{rad},-20 \mu \mathrm{rad},-30 \mu \mathrm{rad}$ tilt angle.

As Fig. 3 showed, when the mirror $M_{1}$ is not tilted, the far-field intensity distribution is Gauss distribution. A sidelobe exits because of the hard-edged diffraction of mirror $M_{2}$. When mirror $M_{1}$ is tilted in anticlockwise direction, the far-field distribution drifted with the beam deflection, but the far-field distribution shape nearly did not change. Curve (c) is the simulated far-field distribution when the mirror $M_{1}$ is tilted $30 \mu \mathrm{rad}$. So the output beam mode is nearly not influenced when mirror $M_{1}$ is tilted with a small angle, only the output beam direction changed. If mirror $M_{1}$ is tilted more and more, as Fig. 4 showed, curve (a) and curve (b) is far-field intensity distribution with $50 \mu \mathrm{rad}$ tilt and $100 \mu \mathrm{rad}$ tilt, respectively. In this obvious tilt situation, the sidelobe effect is more strong. The output mode seems to have some trend of multimode distribution. The beam quality deteriorated but the main part of far-field distribution still remains Gauss distribution. If only considering single-mode output, the far-field intensity distribution influence of mirror $M_{1}$ is tilted in anticlockwise is not sensitive.

As curve (d) showed in Fig. 3, when the tilt angle is $10 \mu \mathrm{rad}$ in clockwise direction, the far-field distribution is 


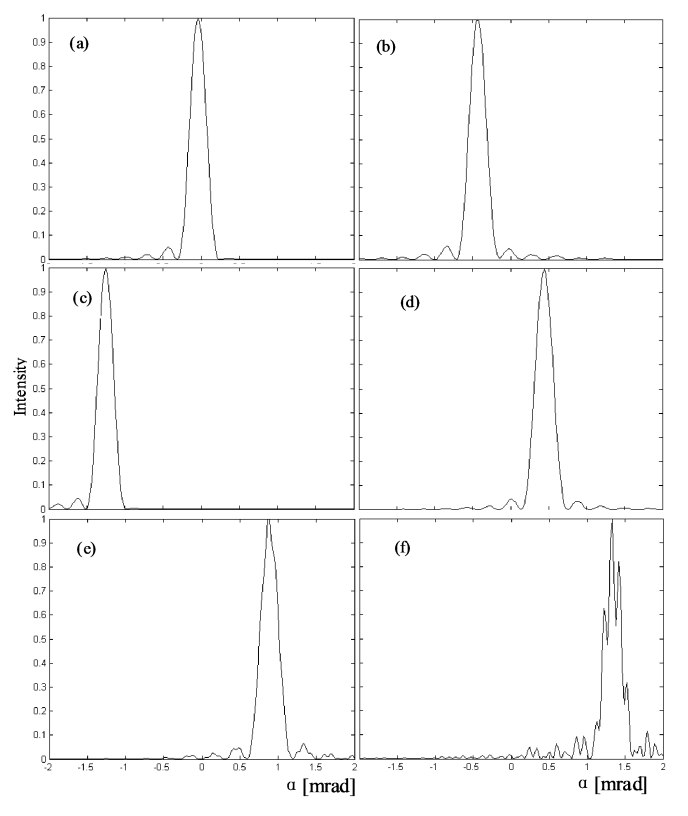

Fig. 3. The far-field distribution curves with different tilt angle $\theta$ of mirror $M_{1}$. "- " means clockwise tilt. (a) $\theta=0 \mu \mathrm{rad}$, (b) $\theta=10 \mu \mathrm{rad}$, (c) $\theta=30 \mu \mathrm{rad}$, (d) $\theta=-10 \mu \mathrm{rad}$, (e) $\theta=-20 \mu \mathrm{rad}$, (f) $\theta=-30 \mu \mathrm{rad}$.

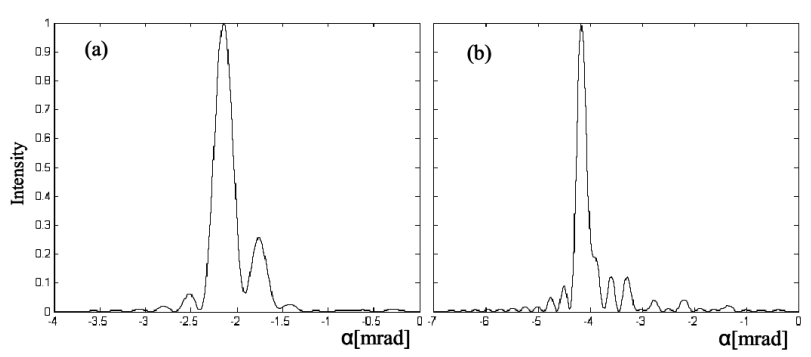

Fig. 4. The far-field distribution curves with big tilt angle in anticlockwise direction (a) $\theta=50 \mu \mathrm{rad}$, (b) $\theta=$ $100 \mu \mathrm{rad}$.

still Gauss distribution. When the tilt angle is $20 \mu \mathrm{rad}$, as curve (e) showed, the far-field distribution curve is mainly Gauss distribution with slight deformation. It seems that there is some trend of multimode distribution. When the tilt angle is $30 \mu \mathrm{rad}$, as curve (f) showed, the far-field distribution is multimode distribution.

Because of the different influence of two directions, beam propagation factor ( $M^{2}$ value) changing is still different in two directions. According to the simulation results, the $M^{2}$ value vs. $M_{1}$ tilt angle is shown in Table. As Table showed, with mirror $M_{1}$ is tilted more in anticlockwise direction, the $M^{2}$ value is getting more and more, with varied value from 1.13 to 1.63 . The beam quality is getting worse. But even the tilted angle is so big (100 $\mu \mathrm{rad})$, the $M^{2}$ value is still small (1.63). So for getting good $M^{2}$ value, the anticlockwise misalignments tolerance of $M_{1}$ is more than $100 \mu \mathrm{rad}$. However, when mirror $M_{1}$ is tilted in clockwise direction more and more, the $M^{2}$ value is less, with the value varied from 1.13 to 1.09. According to this changing trend, the output beam quality seems getting better. If the tilt angle continues increasing, the multimode output is got, as Fig. 3 showed. The clockwise misalignments tolerance of $M_{1}$ mirror is less than $30 \mu \mathrm{rad}$.

TABLE

$M^{2}$ value with mirror $M_{1}$ misalignments.

\begin{tabular}{c|c|c}
\hline $\begin{array}{c}\text { Tilted angle } \\
{[\mu \mathrm{rad}]}\end{array}$ & $\begin{array}{c}M^{2} \text { value with } \\
\text { anticlockwise tilt }\end{array}$ & $\begin{array}{c}M^{2} \text { value with } \\
\text { clockwise tilt }\end{array}$ \\
\hline 0 & 1.13 & 1.13 \\
1 & 1.14 & 1.13 \\
5 & 1.14 & 1.12 \\
10 & 1.15 & 1.12 \\
20 & 1.17 & 1.09 \\
30 & 1.18 & \\
100 & 1.63 &
\end{tabular}

According to the above analysis, the far-field intensity distribution influence of mirror $M_{1}$ tilt is asymmetric in clockwise direction and anticlockwise direction. The misalignments tolerance of anticlockwise direction is bigger than that of clockwise direction. This is different from coaxial unstable resonator. Of course, the misalignments tolerance analysis mainly focused whether the output mode is single mode.

The upper analysis of output beam quality is focused at $M^{2}$ value. In fact, only using $M^{2}$ parameter to evaluate beam quality of unstable resonator is not wholly confident [13]. As we know, unstable resonator is mainly used in high energy laser. Because the energy concentration is more important to high energy laser, power in the bucket (PIB) can be a method for evaluating beam quality.

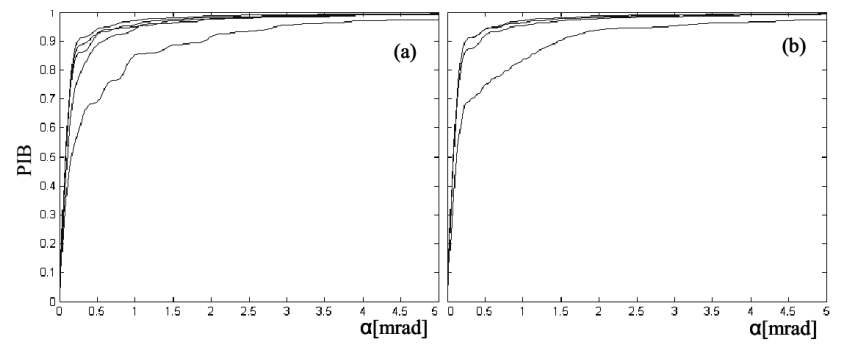

Fig. 5. PIB curves of anticlockwise (a) and clockwise (b) tilt. From the top down, the tilt angle is $0 \mu \mathrm{rad}$, $10 \mu \mathrm{rad}, 20 \mu \mathrm{rad}, 30 \mu \mathrm{rad}, 100 \mu \mathrm{rad}$, respectively.

The PIB curves are also simulated, as Fig. 5 showed. Figure 5a showed the simulated PIB curves when the mirror $M_{1}$ is tilted in anticlockwise direction. The corresponding curves are listed from the top down, respectively with $0 \mu \mathrm{rad}, 10 \mu \mathrm{rad}, 20 \mu \mathrm{rad}, 30 \mu \mathrm{rad}, 100 \mu \mathrm{rad}$ tilt angle. Figure 5b showed the simulated PIB curves when the mirror $M_{1}$ is tilted in clockwise direction. The tilt angle is $0 \mu \mathrm{rad}, 10 \mu \mathrm{rad}, 20 \mu \mathrm{rad}, 30 \mu \mathrm{rad}$, respectively. The corresponding curves are still listed from the top down. 
As Fig. 5a showed, with the mirror $M_{1}$ is tilted more, the energy concentration quality is worse. So the divergence angle of output laser increased and beam quality is worse. This trend is the same as the analysis result of $M^{2}$ value. But in Fig. 5b, the energy concentration quality is worse with increased tilt angle, which means beam quality is also worse. This is contradictory to the situation of decreased $M^{2}$ value. So in clockwise direction, comparing the simulation results of $M^{2}$ value and PIB curves, the varied beam quality trend is consistent, but not in anticlockwise direction. Evaluating beam quality only by $M^{2}$ value is not reasonable for off-axial unstable resonator.

\section{Conclusion}

The three-dimensional and two-dimensional output far-field distributions of off-axial cylindrical hybrid resonator are simulated in this paper. The simulations are well matched with experiments results. The influences of mirror misalignments of off-axial hybrid resonator are well analyzed, including the asymmetric misalignments tolerance and beam quality analysis. It demonstrated that the beam quality evaluation of off-axial unstable resonator should not be one-sided. For high power laser, beam quality evaluating should be considered by the actual situation.

\section{Acknowledgments}

The authors acknowledge the support of Shandong Provincial Natural Science Foundation (ZR2010DQ015), China, the Fundamental Research Funds for the Central Universities (14CX02023A, 14CX02021A), and Basic Research Project of Qingdao Science and Technology Plan (13-1-4-225-jch).

\section{References}

[1] P.E. Jackson, H.J. Baker, D.R. Hall, Appl. Phys. Lett. 54, 1950 (1989).

[2] A. Lapucci, A. Labate, F. Rossetti, S. Mascalchi, Appl. Opt. 35, 3185 (1996).

[3] K.M. Du, N.L. Wu, J.D. Xu, J. Giesekus, P. Loosen, R. Poprawe, Opt. Lett. 23, 370 (1998).

[4] M. Ciofini, E. Favilla, A. Lapucci, E. Sani, Opt. Laser Technol. 39, 1380 (2007).

[5] K.M. Du, D.J. Li, H.L. Zhang, P. Shi, X.Y. Wei, R. Diart, Opt. Lett. 28, 87 (2003).

[6] P. Shi, D.J. Li, H.L. Zhang, Y.D. Wang, K.M. Du, Opt. Commun. 229, 349 (2004).

[7] H.L. Zhang, P. Shi, D.J. Li, K.M. Du, Appl. Opt. 42, 1681 (2003).

[8] E.A. Sziklas, A.E. Siegman, Appl. Opt. 14, 1874 (1975).

[9] N. Wang, P. Shi, Y.T. Lu, Proc. SPIE 5627, 357 (2005).

[10] A.E. Siegman, Lasers, University Science Books, Mill Valley Calif. 1986, Ch. 22

[11] Z. Wang, X. Ye, T. Fang, J. Xu, Opt. Rev. 12, 391 (2005).

[12] N. Wang, Ph. D. Thesis, Shanghai Institute of Optics and Fine Mechanics, Chinese Academy of Sciences, 2005 .

[13] A.E. Siegman, Opt. Soc. Am. Top. 17, 184 (1998). 